American Medical Journal 3 (2): 93-99, 2012

ISSN 1949-0070

(C) 2012 Science Publications

\title{
Insulin Pump Therapy in Type 1 Diabetes-Merging Technology with Diabetes Care
}

\author{
Thabit, $\mathrm{H}$. \\ Department of Endocrinology, Wolfson Diabetes and Endocrine Clinic, \\ Addenbrookes Hospital, Cambridge University Hospitals, Cambridge, United Kingdom
}

\begin{abstract}
The use of insulin pump therapy has been increasing over the past number of years in many countries. The Diabetes Control and Complications Trial (DCCT) have proven that better glycaemic control with fewer hypoglycemic events is achievable with insulin pump therapy. The delivery of subcutaneous insulin through an insulin pump mimics physiological insulin delivery better than Multiple Daily Injection of insulin (MDI). Insulin pump therapy is an attractive therapeutic option for many patients with T1DM with the potential to improve glycaemic control and quality of life. Technological developments over the past few years have enhanced the functional capabilities and appearance of modern insulin pumps. Careful patient selection for insulin pump therapy by health care professionals is still important, to optimize its benefit and to lessen the associated risk. In this review article, the clinical evidence for insulin pumps, its advantages and disadvantages, as well as patient selection criteria for insulin pumps are outlined. Examples of insulin pump devices that are currently available, including the advent of newer devices and technology in this field are also reviewed.
\end{abstract}

Key words: Diabetes Control and Complications Trial (DCCT), Multiple Daily Injection of insulin (MDI), Continuous Subcutaneous Insulin Infusion (CSII)

\section{INTRODUCTION}

The importance of glycaemic control in type 1 diabetes has previously been established by the Diabetes Control and Complications Trial (DCCT) (NIS, 1993). In the DCCT, tight glycaemic control using either Multiple Daily Injection of insulin (MDI) or insulin pumps dramatically reduced the prevalence of microvascular complications and has since significantly changed the standard goal of therapy for T1DM. In this study, patients who were treated with insulin pumps, also known as Continuous Subcutaneous Insulin Infusion (CSII) therapy had moderately better glycaemic control and fewer hypoglycemic events. This and other studies have since rekindled interest in the applications of CSII therapy in T1DM patients.

The first insulin pump was introduced by a US physician Kadish (1964). The prototype that he designed however had to be worn as a backpack (Fig. 1). It was also heavy and cumbersome, making it impractical for daily use. Compared to the insulin analogues that are currently used in clinical practice today, the insulin used in insulin pumps at the time suffered from having unpredictable peaks and troughs. This made hypoglycemia much more common and difficult to manage. It was not until the publication of the DCCT and the involvement of pharmaceutical manufacturers such as Medtronic, Roche and Abbot, that CSII therapy came back into mainstream clinical practice. These modern pump devices are smaller, more durable and easier to use, compared to their early predecessors. Data from industry estimates that there are currently 200,000-250,000 patients on CSII in the United States (Selam, 2006). In continental Europe, such as Germany, around $10 \%$ of patients with T1DM are on CSII therapy (Renard, 2010; Selam, 2006; Haugstvedt et $a l ., 2010)$. This figure is even smaller in the UK and is thought by many diabetologists to be an underutilised therapeutic option for T1DM.

In this review article, the clinical evidence for CSII, its advantages and disadvantages, as well as patient selection criteria for CSII are outlined. We will also give examples of insulin pump devices that are currently available, including the advent of newer devices and technology in this field.

\section{The principles and advantages of CSII therapy:} The insulin pump device consists of a subcutaneous infusion set (i.e.; cannula and tubing system), a reservoir of insulin (within the pump) and the pump itself. The infusion set is disposable and needs to be changed every 2-3 days. The insulin used in CSII devices is commonly rapid acting analogues such as asphalt, lispro and glulisine. 


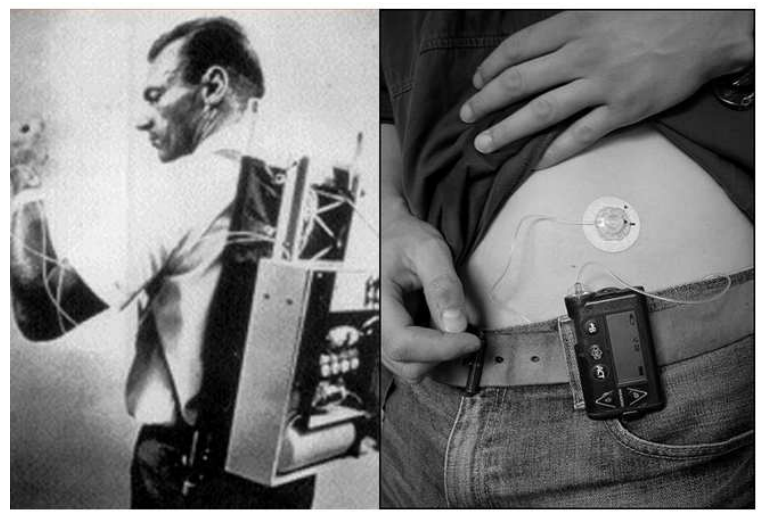

Fig. 1: The first insulin pump prototype was designed by Dr. Kadish (left) compared to a modern insulin pump device (right)

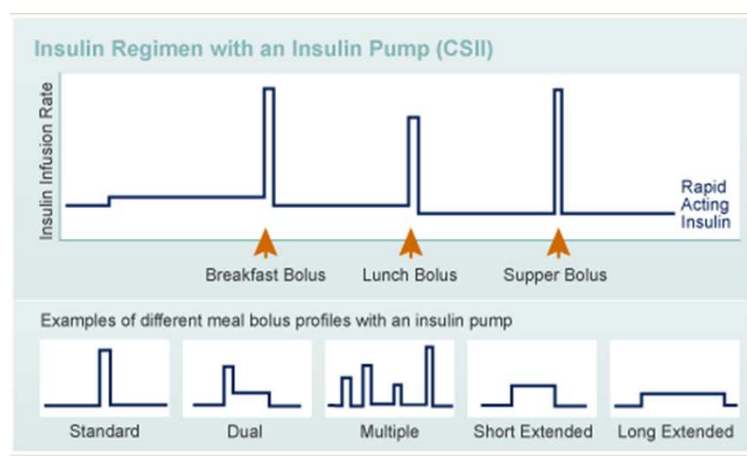

Fig. 2: Example of insulin delivery with pump therapy. By using a single type of rapid acting insulin, basal rates can be programmed to match individual patient's basal insulin requirements (i.e.; higher basal rates for early morning hours to limit hyperglycemia caused by the dawn phenomenon). Bolus insulin during meals can be delivered either as an immediate/standard bolus (in-between meal snacks) or extended bolus (for larger meals and to mimic the physiological second phase insulin response)

The insulin pump is programmed to deliver rapid acting insulin in two different ways; a slow continuous and adjustable infusion rate over $24 \mathrm{~h}$ (basal rate) and a single bolus dose that can be given at mealtimes or to correct hyperglycemia (Fig. 2). Modern pumps are sometimes known as smart pumps, as they have a builtin mealtime customizable calculator device, known as "Bolus Wizards" (Zisser et al., 2008). This allows the user to input mealtime carbohydrate load. In addition the Wizard also factors in the most recent glucometer reading and the amount of Insulin On Board (IOB). The
"Bolus Wizard" then advises the user on appropriate premeal insulin bolus dose based on the patient's planned carbohydrate intake and IOB. This not only allows for greater accuracy, but by discounting the residual insulin that is still around, it avoids stacking of insulin doses and therefore reduces the risk of hypoglycemia (Shashaj et al., 2008).

Figure 2 Example of insulin delivery with pump therapy. By using a single type of rapid acting insulin, basal rates can be programmed to match individual patient's basal insulin requirements (i.e.; higher basal rates for early morning hours to limit hyperglycemia caused by the dawn phenomenon). Bolus insulin during meals can be delivered either as an immediate/standard bolus (in-between meal snacks) or extended bolus (for larger meals and to mimic the physiological second phase insulin response)

There are several advantages of CSII therapy (listed in Table 1):

Better glycaemic control and variability: Earlier studies have shown that CSII provides as good and often better glycaemic control when compared to conventional MDI therapy (Hanaire-Broutin et al., 2000). CSII therapy also has the advantage of mimicking physiological insulin secretion better than MDI therapy due to the variable rate of basal insulin that can be infused. This reduces glycaemic variability, resulting in less blood glucose fluctuations throughout the day (Pickup and Keen, 2002). The 5-Nations Trial, which is one of the largest randomized controlled trial of CSII therapy, has shown that CSII is superior to MDI in lessening overall blood glucose fluctuations (mean $0.8 \mathrm{mmol} / \mathrm{L}$ ) and also led to better Quality Of Life (QOL) scores (Hoogma et al., 2006).

A study published in the New England Journal of Medicine, known as the STAR3 Study, compared the efficacy of sensor-augmented CSII therapy with MDI in children and adults (Bergenstal et al., 2010). A greater proportion of patients on the pump therapy achieved lower HbA1c levels $(7.5 \%$ vs. $8.1 \%$, p $<0.001)$, without increased incidence of hypoglycemia. This study therefore illustrates that as pump technology continues to improve, tighter glycaemic control can potentially be achieved safely in patients with T1DM.

Table 1: List of advantages and disadvantages of CSII therapy

\begin{tabular}{ll}
\hline Advantages & Disadvantages \\
\hline Better glycaemic control without & Frequent monitoring of \\
The increased risk of hypoglycaemia & blood glucose needed \\
Mimics physiological insulin secretion, & Risk of diabetic ketoacidosis if \\
therefore reduces glycaemic & insulin delivery is interrupted \\
variability /fluctuations & (i.e.; catheter occlusion, pump failure) \\
Reduced risk of nocturnal hypoglycaemia & Catheter-site infection \\
Greater lifestyle flexibility for patients & Ready access to MDI still needed \\
& in case of pump failure \\
\hline
\end{tabular}


Reduced risk of hypoglycemia: hypoglycemia is a significant problem faced by patients on insulin therapy, particularly at night time (Ahmet et al., 2011). In the DCCT, intensified treatment was associated with a 3-fold increased risk of hypoglycemia events. In the same study however, those on CSII in the intensively treated group experienced less hypoglycemic events than their MDI counterparts.

A greater number of studies have since proven that CSII is superior to MDI in reducing hypoglycemic risk (Boland et al., 1999; Pickup and Keen, 2002; Pickup et al., 2006).

More importantly, CSII have been beneficial in reducing the frequency of nocturnal hypoglycemia. It is reported that up to $50 \%$ of hypoglycemia occur at night (Buckingham et al., 2008). This is of significant concern, especially among parents of children with T1DM. In prospective randomized studies, the incidence of nocturnal hypoglycemia and glucose level variability was decreased significantly (Pickup and Keen, 2002). This is due to the ability of CSII to deliver insulin at night with more physiological pharmacokinetics and the ability to adjust the infusion rate appropriately and safely in patients who have higher early morning blood glucose levels. Parents also report greater satisfaction with CSII therapy, as the infusion rate can be adjusted according to their children's earlier daytime activities or meal times (Muller-Godeffroy et al, 2009).

Better lifestyle flexibility: One of the main benefits cited by patients who have chosen CSII therapy is the degree of flexibility that CSII can offer on their lifestyle (Todres et al., 2010). It allows patients to modify their insulin infusion rate by the hour, tailoring it according to their activity or performance during the day (i.e.; exercise, delaying meals, sleeping in late on weekends). Another benefit is the convenience of not having to give multiple injections per day. However, patients on CSII therapy are still advised to check their blood glucose several times per day and have ready access to MDI therapy, in case of pump failure.

Disadvantages of CSII: Despite the increasing awareness and use of CSII therapy, disadvantages of a pump therapy is worthy of discussion as well.

Firstly, current CSII therapy is not an artificial pancreas. Patients will still need to monitor their blood glucose levels, at least 4 times daily and usually more. While it is true the latest pumps have smart technology systems (i.e.; Bolus Wizard), it is still dependent on the user's input of information and data into the system.
Therefore, patients still have to pay particular attention to their blood glucose measurements, carbohydrate intake and hyper/hypo-glycaemia corrections.

Secondly, CSII therapy does not completely eliminate episodes of hypo- or hyperglycemia. Although there is now strong evidence that CSII can provide better glycaemic control with reduced glycaemic variability, it is still at risk of technical faults (Guilhem et al, 2006). As there is no subcutaneous depot of long-acting insulin in CSII, any interruption of insulin delivery puts the patient at risk of rapid onset diabetic ketoacidosis, especially if the interruption is prolonged. This can be caused by catheter displacement, catheter/tubing occlusion, battery failure and depletion of insulin supply. Hypoglycemia is less common.

Newer pumps have built-in hypoglycemia alarm that alerts the patient if their blood glucose is going low, although the phenomenon of "alarm fatigue" have been reported, causing patients to unintentionally ignore their alarm warnings if occur too often (Buckingham et al., 2010). Regular blood glucose measurements are therefore still very important, in order to inform the patient of impending hypo- or hyperglycemia, thereby allowing them to take the necessary precaution or action.

One misconception is that patients no longer need to take insulin injections. All patients on CSII therapy must be educated to give MDI therapy in case of pump failure, or if they pump had to be disconnected for a prolonged period of time for any particular reason (i.e., Hospitalization for acute illness, surgery). Therefore these patients should have access and the capacity to selfadminister subcutaneous insulin injections if needed.

A complication sometimes associated with CSII therapy is catheter-site infection (Mecklenburg, 1989). The annual occurrence is estimated to be around 7-11 events per 100 years of patient follow-up. Most catheter-site infections are caused by Staphylococcus or Streptococcus species. In very rare cases, severe infections can lead to cellulitis or abscess requiring surgical drainage. Patients should always be advised to change their catheter site every 2 or 3 days to minimize risk of developing skin infections.

Patient selection and indication for CSII therapy: It is important that physicians intending to select their patients in CSII therapy are aware of the indications and selection criteria, in order to optimize the benefits gained and lessen their associated risks. There are several professional bodies currently defining the indications for CSII, based on their merits and safety.

In 2010, the American Association of Clinical Endocrinologist issued a joint statement, guiding clinicians on exclusion criteria for CSII therapy 
(Grunberger et al., 2010). This included patients who are unable or unwilling to perform frequent blood glucose testing, carbohydrate counting and multiple daily injections. Patients with history of non-adherence to insulin injections, poor motivation or unrealistic expectations of pump therapy are also not suitable candidates for CSII therapy. Any history of serious psychological or psychiatric conditions should also be an exclusion criterion for initiating CSII, as the risk of poor compliance and adverse events on CSII therapy is significant. This joint statement however was produced not only to guide appropriate patient selection, but also to ensure that the clinician is competent in CSII therapy and management and takes responsibility of instigating a comprehensive pump management program in a multi-disciplinary setting for patients on this complex diabetes therapy. In the United States there is currently no official requirement for medical supervision of CSII therapy or a certifying process to ensure that clinicians are qualified to prescribe CSII therapy. Adverse outcomes have been reported in patients who were left with inadequate training and expert guidance on CSII therapy. The hope is that by having guidelines both for patients and clinicians, these unfortunate events can be minimized.

The NICE guidelines in the UK currently recommend CSII in patients who are unable to achieve target HbA1c levels without experiencing disabling hypoglycemia (repeated and unpredictable hypoglycemia associated with significant adverse effect on quality of life). They also recommend CSII therapy in T1DM patients, who despite receiving a high level of care and input, still have suboptimal HbA1c levels (at $8.5 \%$ or above) on MDI therapy (Cummins et al., 2010). In these patients, CSII can be continued if they demonstrate an improvement in glycaemic control, or a decrease in the rate of hypoglycemic episodes.

It is important that clinicians carefully explain to patients the proper indications for CSII therapy, as well as to the reasons why they might not be suitable for the same. If used correctly, CSII therapy has the potential to improve not only glycaemic and metabolic control, but also the patient's overall quality of life and satisfaction with their diabetes care.

Recent developments in insulin pump technology: Technological advances in recent years have allowed the development of more sophisticated CSII devices with enhanced features. Frequent self-monitoring of blood glucose still remains the foundation for optimal CSII therapy. However, CGM use is currently gaining more attention as it allows further insight and information into glycaemic pattern and variability. One of the developers of insulin pump technology, Medtronic, has developed a sensor-augmented insulin pump known as the MiniMed Paradigm Veo® (Medtronic MiniMed, Northridge, CA, USA), which integrates CSII with real-time CGM sensor (Fig. 3). The CGM component of the system allows continuous monitoring of blood glucose, as well as giving the patient predictive alerts of oncoming hyper or hypoglycemia, based on the trend of blood glucose levels. This information allows the patient to adjust the rate of insulin delivery from the pump in an earlier and safer manner. The efficacy and clinical utilization of this technology have been established in the STAR3 study in which better glycaemic control was achieved safely in CSII therapy compared to MDI (Slover et al., 2012).

The Low Glucose Suspend (LGS) function by the Medtronic Paradigm Veo pump is another recent development in insulin pump technology. When hypoglycemia is detected by the integrated CGM and the hypoglycemia alarm is not acknowledged by the patient, the insulin pump automatically suspends insulin delivery for up to $2 \mathrm{~h}$. The aim of LGS is to mitigate the risk of hypoglycemia, especially at night-time, when patients are at greater risk of seizures from prolonged low sensor glucose levels. Post-marketing studies proved that LGS function significantly reduces the duration of nocturnal hypoglycemia, without the risk of significant rebound hyperglycemia (median sensor glucose $8.2 \mathrm{mmol} / 1$ 2-hours after basal insulin restarts) (Choudhary et al., 2011; Danne et al., 2011). Patients also reported greater satisfaction and reassurance when utilizing the LGS function.

The size and design of modern insulin pumps have undergone significant changes in recent years. Patch pumps are "tubingless" insulin pumps, in which the insulin reservoir and infusion set system are housed in the same unit. An example of the patch pump is the Omnipod® (Insulet, MA, USA), Fig. 4. The Omnipod ${ }^{\circledR}$ insulin pump system consists of a disposable infusion pump (also known as a Pod) that is typically changed every $72 \mathrm{~h}$ and a Personal Diabetes Manager (PDM) which remotely controls the pump. The advantage of the patch pump is that it is tubingless, making it smaller and more discrete compared to conventional insulin pumps. This may be an important factor in patients who may otherwise reject pump therapy due to the physical appearance and obstructive nature of external tubing. Patch pumps are also less affected by the hydrostatic or 'siphon' effect, i.e.; overor under-delivery of insulin delivery, due to movement of the insulin pump in relation to infusion site (Zisser et $a l ., 2010)$. The clinical significance of the 'siphon' effect however is currently unknown. 


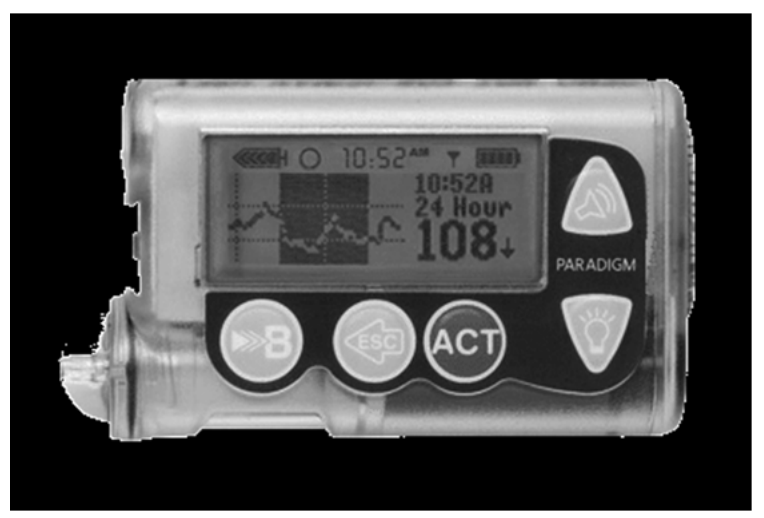

Fig. 3: An example of a sensor augmented insulin pump, the Medtronic Paradigm Veo ${ }^{\circledR}$

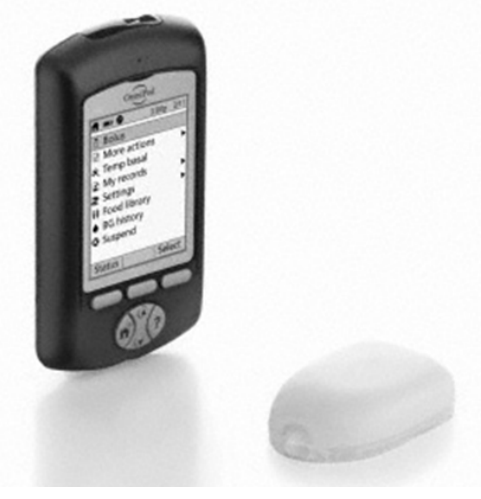

Fig. 4: An example of a patch pump, the Omnipod $囚$. Shown here is the disposable infusion pump (Pod) and the Personal Diabetes Manager (PDM)

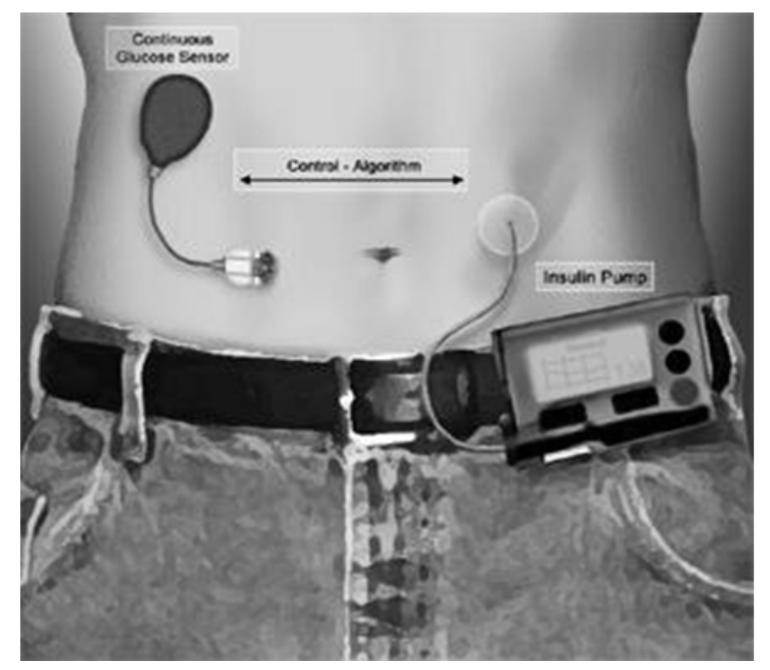

Fig. 5: An illustrated example of a closed-loop system, using an automated glucoregulatory feedback mechanism
The ultimate goal in CSII technology is the development of a closed-loop, or so called 'artificial pancreas' system (Fig. 5). A true closed-loop system is one that mimics the physiology of the pancreas, by linking continuous glucose measurements with automated delivery of insulin to normalize blood glucose levels under the control of an automated computer algorithm, with minimal patient intervention (Hovorka, 2011; Steil et al., 2004). Research and development into this technology is currently underway. Although there are still several roadblocks before a true 'artificial pancreas' is available, the next few years will certainly be exciting in this field. This may ultimately revolutionize the delivery of T1DM care in the future.

\section{REFERENCES}

Ahmet, A., S. Dagenais, N.J. Barrowman, C.J. Collins and M.L. Lawson et al., 2011. Prevalence of nocturnal hypoglycemia in pediatric type 1 diabetes: A pilot study using continuous glucose monitoring. J. Pediatr., 159: 297-302. DOI: 10.1016/j.jpeds.2011.01.064

Bergenstal, R.M., W.V. Tamborlane, A. Ahmann, J.B. Buse and G. Dailey et al., 2010. Effectiveness of sensor-augmented insulin-pump therapy in type 1 diabetes. N Engl. J. Med., 363: 311-320. DOI: 10.1056/NEJMoa1002853

Boland, E.A., M. Grey, A. Oesterle, L. Fredrickson and W.V. Tamborlane, 1999. Continuous subcutaneous insulin infusion. A new way to lower risk of severe hypoglycemia, improve metabolic control and enhance coping in adolescents with type 1 diabetes. Diabetes Care, 22: 1779-1784. DOI: 10.2337/diacare.22.11.1779

Buckingham, B., D.M. Wilson, T. Lecher, R. Hanas and K. Kaiserman et al., 2008. Duration of nocturnal hypoglycemia before seizures. Diabetes Care, 31: 2110-2112. DOI: 10.2337/dc08-0863

Buckingham, B., H.P. Chase, E. Dassau, E. Cobry and P. Clinton et al., 2010. Prevention of nocturnal hypoglycemia using predictive alarm algorithms and insulin pump suspension. Diabetes Care, 33: 1013-1017. DOI: 10.2337/dc09-2303

Choudhary, P., J. Shin, Y. Wang, M.L. Evans and P.J. Hammond et al., 2011. Insulin pump therapy with automated insulin suspension in response to hypoglycemia: Reduction in nocturnal hypoglycemia in those at greatest risk. Diabetes Care, 34: 2023-2025. DOI: 10.2337/dc10-2411 
Cummins, E., P. Royle, A. Snaith, L. Robertson and L. McIntyre et al., 2010. Clinical effectiveness and cost-effectiveness of continuous subcutaneous insulin infusion for diabetes: Systematic review and economic evaluation. Health Technol. Assess., 14: 1-181. PMID: 20223123

Danne, T., O. Kordonouri, M. Holder, H. Haberland and S. Golembowski et al., 2011. Prevention of hypoglycemia by using Low Glucose Suspend function in sensor-augmented pump therapy. Diabetes Technol. Ther., 13: 1129-1134. DOI: 10.1089/dia.2011.0084

Grunberger, G., T.S. Bailey, A.J. Cohen, T.M. Flood and Y. Handelsman et al., 2010. Statement by the American association of clinical endocrinologists consensus panel on insulin pump management. Endocr. Pract., 16: 746-762. PMID: 21356638

Guilhem, I., A.M. Leguerrier, F. Lecordier, J.Y. Poirier and D. Maugendre, 2006. Technical risks with subcutaneous insulin infusion. Diabetes Metab., 32: 279-284. DOI: 10.1016/S1262-3636(07)70281-1

Hanaire-Broutin, H., V. Melki, S. Bessieres-Lacombe and J.P. Tauber, 2000. Comparison of continuous subcutaneous insulin infusion and multiple daily injection regimens using insulin lispro in type 1 diabetic patients on intensified treatment: A randomized study. The study group for the development of pump therapy in diabetes. Diabetes Care, 23: 1232-1235. DOI: 10.2337/diacare.23.9.1232

Haugstvedt, A., T. Wentzel-Larsen, M. Graue, O. Søvik and B. Rokne, 2010. Fear of hypoglycaemia in mothers and fathers of children with Type 1 diabetes is associated with poor glycaemic control and parental emotional distress: A populationbased study. Diabet Med., 27: 72-78. DOI: 10.1111/j.1464-5491.2009.02867.x

Hoogma, R.P.L.M., P.J. Hammond, R. Gomis, D. Kerr and D. Bruttomesso et al., 2006. Comparison of the effects of Continuous Subcutaneous Insulin Infusion (CSII) and NPH-based Multiple Daily Insulin injections (MDI) on glycaemic control and quality of life: Results of the 5-nations trial. Diabet Med., 23: 141-147. DOI: 10.1111/j.14645491.2005.01738.x

Hovorka, R., 2011. Closed-loop insulin delivery: From bench to clinical practice. Nat. Rev. Endocrinol., 7: 385-395. DOI: 10.1038/nrendo.2011.32

Kadish, A.H., 1964. Automation control of blood sugar. I. A servomechanism for glucose monitoring and control. Am. J. Med. Electron., 3: 82-86. PMID: 14150660
Mecklenburg, R.S., 1989. Acute complications associated with the use of insulin infusion pumps. Diabetes Educ., 15: 40-43. DOI: 10.1177/014572178901500111

Muller-Godeffroy, E., S. Treichel and V.M. Wagner, 2009. Investigation of quality of life and family burden issues during insulin pump therapy in children with Type 1 diabetes mellitus-a largescale multicentre pilot study. Diabet Med., 26: 493501. DOI: 10.1111/j.1464-5491.2009.02707.x

NIS, 1993. The effect of intensive treatment of diabetes on the development and progression of long-term complications in insulin-dependent diabetes mellitus. The diabetes control and complications trial research groupn. Engl. J. Med., 329: 977-986. PMID: 8366922

Pickup, J. and H. Keen, 2002. Continuous subcutaneous insulin infusion at 25 years: Evidence base for the expanding use of insulin pump therapy in type 1 diabetes. Diabetes Care, 25: 593-598. DOI: 10.2337/diacare.25.3.593

Pickup, J.C., J. Kidd, S. Burmiston and N. Yemane, 2006. Determinants of glycaemic control in type 1 diabetes during intensified therapy with multiple daily insulin injections or continuous subcutaneous insulin infusion: importance of blood glucose variability. Diabetes Metab. Res. Rev., 22: 232237. DOI: $10.1002 /$ dmrr.614

Renard, E., 2010. Insulin pump use in Europe. Diabetes Technol. Ther., 12: S29-S32. DOI: 10.1089/dia.2009.0189

Selam, J.L., 2006. CSII in Europe: Where are we, where are we going? An analysis of articles published in infusystems international. Diabetes Res. Clin. Pract., 74: S123-S126. DOI: 10.1016/S0168-8227(06)70014-6

Shashaj, B., E. Busetto and N. Sulli, 2008. Benefits of a bolus calculator in pre- and postprandial glycaemic control and meal flexibility of paediatric patients using Continuous Subcutaneous Insulin Infusion (CSII). Diabet Med., 25: 1036-1042. DOI: 10.1111/j.1464-5491.2008.02549.x

Slover, R.H., J.B. Welsh, A. Criego, S.A. Weinzimer and S.M. Willi et al., 2012. Effectiveness of sensor-augmented pump therapy in children and adolescents with type 1 diabetes in the STAR 3 study. Pediatr Diabetes, 13: 6-11. DOI: 10.1111/j.1399-5448.2011.00793.x

Steil, G.M., A.E. Panteleon and K. Rebrin, 2004. Closed-loop insulin delivery-the path to physiological glucose control. Adv. Drug. Deliv. Rev., 56: 125-144. DOI: 10.1016/j.addr.2003.08.011 
Todres, L., S. Keen and D. Kerr, 2010. Continuous subcutaneous insulin infusion in Type 1 diabetes: patient experiences of 'living with a machine'. Diabet Med., 27: 1201-1204. DOI: 10.1111/j.14645491.2010.03058.x

Zisser, H., L. Robinson, W. Bevier, E. Dassau and C. Ellingsen et al., 2008. Bolus calculator: A review of four "smart" insulin pumps. Diabetes Technol. Ther., 10: 441-444. DOI: 10.1089/dia.2007.0284
Zisser, H.C., W. Bevier, E. Dassau and L. Jovanovic, 2010. Siphon effects on continuous subcutaneous insulin infusion pump delivery performance. J. Diabetes Sci. Technol., 4: 98-103. PMID: 20167172 